\title{
Research Article \\ Traffic Optimization at Junctions to Improve Vehicular Flows
}

\author{
A. Cutolo, C. D'Apice, and R. Manzo \\ Dipartimento di Ingegneria Elettronica e Ingegneria Informatica, University of Salerno, Via Ponte don \\ Melillo, 84084 Fisciano (SA), Italy \\ Correspondence should be addressed to R. Manzo, rmanzo@unisa.it
}

Received 14 April 2011; Accepted 22 May 2011

Academic Editor: E. J. Sellountos

Copyright ( $) 2011$ A. Cutolo et al. This is an open access article distributed under the Creative Commons Attribution License, which permits unrestricted use, distribution, and reproduction in any medium, provided the original work is properly cited.

\begin{abstract}
The aim of this work is to improve urban traffic viability through an appropriate choice of yielding and stop signs or red and green phases for traffic lights in junctions with two entering and one exiting roads (junctions of $2 \times 1$ type). We consider a macroscopic fluid-dynamic model able to capture the traffic evolution. We analyze different functionals measuring networks performance in terms of average velocity, average traveling time, total flux, density, stop and go waves, average traveling time, weighted with the number of cars moving on roads, and kinetic energy. Right of way parameters which optimize the latter two functionals are obtained. Simulations of simple junctions of $2 \times 1$ type have been used to test the correctness of the analytical results. Then, global performance of optimization procedures has been investigated on Re di Roma Square, in Italy. In particular, we discuss cases in which the functionals are optimized locally at each junction for different values of right of way parameters. We show that for the chosen initial data the only algorithm for the maximization of velocity assures globally the best performance for the network, also in terms of average traveling times and kinetic energy.
\end{abstract}

\section{Introduction}

The problem of traffic modeling is a very huge task, due to the complexity of the system to analyze. Many methods have been developed based on different approaches ranging from microscopic one, taking into account each single car, to kinetic and macroscopic ones, dealing with averaged quantities. Of course the understanding and control of traffic phenomena by means of simulation and optimization studies can be useful to eventually make decisions which may alleviate congestion, maximize flow traffic, reduce accidents, and other desirable ends.

Here rather than analyzing the movement of individual cars, we describe road networks behaviour using fluid-dynamic models; hence we treat traffic situations resulting from complex interaction of many vehicles. In particular, following [1] we determine the 
evolution of car traffic on each road with the first-order model of Lighthill, Whitham, and Richards (LWR model), which is on one side simple enough to permit a complete understanding of traffic flows and on the other side rich enough to detect important phenomena as queue formation. A key role in the networks modeling is played by the junctions. In order to capture the dynamics at a node, Cauchy problems with initial data constant on each road, called Riemann Problems at the node, have to be solved. The latter have a unique solution if we introduce some rules:

(A) the cars flow from an incoming road to outgoing ones according to the final destination;

(B) the number of cars passing the junction is maximized respecting rule (A).

For a junction of $1 \times 2$ type, that is, with one incoming road, labeled with 1 , and two outgoing roads, labeled with 2,3, the rule (A) is expressed by a coefficient $\alpha$ which describes the percentage of cars going from road 1 to road 2. Obviously $1-\alpha$ is the percentage of cars moving towards the outgoing road 3.

If the junction has two incoming roads, labeled with 1 and 2 , and one outgoing road, labeled with 3, (junction of $2 \times 1$ type), in order to solve the dynamics, we have to introduce the right of way parameter $p$. Supposing that $C$ cars can enter into the junction, then $p C$ cars come from road 1 and $(1-p) C$ from road 2.

Assigning the initial density of all incoming and outgoing roads in a node, we compute the final equilibrium as function of the traffic distribution coefficient or/and of the right of way parameter depending on junction type. Such equilibrium, belonging to the admissible region for the final fluxes, is chosen according to a fixed strategy, and represents the solution of the Riemann Problem.

The aim of this work is the optimization of urban networks performance with junctions of $2 \times 1$ type through a suitable choice of the right of way parameter $p$. Observe that the choice of $p$ corresponds to the use of yielding and stop signs or to the regulation of red and green phases for traffic lights.

Optimization problems for fluid-dynamic models have been already considered for car traffic: [2] is devoted to traffic light regulation, while [3] and [4] are more related to our analysis but focus on the case of smooth solutions (not developing shocks) and boundary control. A specific traffic regulation problem is addressed in [5]. Given a crossing with some expected traffic, is it preferable to construct a traffic circle or a light? The two solutions are studied in terms of flow control and the performances are compared. In [6-8], the traffic behaviour has been analyzed using four cost functionals, $J_{1}, J_{2}, J_{3}$, and $J_{5}$, measuring, respectively, cars average velocity, cars average traveling time, car velocity, weighted with cars quantity, traveling on the roads, that is, fluxes and stop and go waves (see also [9]). The optimization was done over right of way parameters and traffic distribution coefficients with the aim of maximizing $J_{1}$ and $J_{3}$ and minimizing $J_{2}$. In particular, two special cases of junctions have been considered for the optimization: the $2 \times 1$ and $1 \times 2$ cases.

In this work, we analyze the urban traffic behaviour introducing new functionals. From the solution of the Riemann Problem, we determine the average speeds at which drivers travel and we define additional functionals: $J_{4}$ which measures the density, $J_{6}$ the average traveling time, seen as the sum of the average traveling times on each road, weighted with the number of cars moving on it, and $J_{7}$ which gives information about the kinetic energy. Of course the aim is to optimize the choice of the parameters in order to minimize $J_{6}$ and to maximize $J_{7}$. Since in case of $1 \times 2$ junctions, the functionals $J_{6}$ and $J_{7}$ are optimized for the same values of the distribution coefficients which maximize and minimize $J_{1}$ and $J_{2}$, we 
focused our attention on the optimization of junctions of type $2 \times 1$, for which $J_{1}$ and $J_{6}$ have, in some cases, different optimal values. It is interesting to notice that in many cases (with the extreme case of functionals not depending on $p$ ) there is a set of optimal values of the right of way parameters.

The correctness of analytical optimization algorithms is tested through simulations. First we consider simple junctions of $2 \times 1$ type and compare different choices of parameters, statistical parameters, and optimal one. In particular, the initial density values on the roads are chosen in such way that optimization procedures, obtained evaluating the described cost functionals, give different optimal parameters.

Then, we studied the effects of the decentralized approach on the global performance of more complicated networks. According to this approach local optimal parameters at every junction of a complex network have been used. The discussed example regards Re di Roma Square, in Rome. We notice that the global effect of optimization is achieved using the optimal values for the velocity functional. Indeed, it is also shown that, considering the optimal values for the other cost functionals, the network becomes almost completely full, with consequent birth of congestion phenomena and reduction of cars fluxes. Finally we compare optimal algorithms with random ones. In the latter case, the right of way parameters are chosen randomly at every instant of time and for every junction independently. We see that the optimal algorithm based on the maximization of the velocity ensures better performance than random simulation.

The paper is organized as follows. In Section 2, we recall the basic definitions and the construction of solutions to Riemann Problems at junctions. The subsequent section is devoted to the introduction of the cost functionals and to the optimization of $J_{6}$ and $J_{7}$, compared with $J_{1}$ and $J_{2}$. Then, Section 4 reports simulation results first for simple junctions and then for Re di Roma Square.

\section{Road Network Model}

We consider a traffic network that is a finite collection of roads connected together by junctions. Formally, we introduce the following definition.

Definition 2.1. A traffic network is given by a 4-tuple $(N, \supset, \mathcal{F}, 2)$ where

\section{Cardinality}

$N$ is the cardinality of the network, that is, the number of roads in the network;

Lines

$\supset$ is the collection of roads, modeled by intervals $I_{k}=\left[a_{k}, b_{k}\right] \subseteq \mathbb{R}, k=1, \ldots, N$;

\section{Fluxes}

$\mathcal{F}$ is the collection of flux functions $f_{k}:\left[0, \rho_{k}^{\max }\right] \mapsto \mathbb{R}, k=1, \ldots, N$, with $\rho_{k}^{\max }$ the maximal density on road $I_{k}$; 
Nodes

2 is a collection of subsets of $\{ \pm 1, \ldots, \pm N\}$ representing junctions. If $j \in J \in 2$, then the road $I_{|j|}$ is crossing at $J$ as incoming road (i.e., at point $b_{i}$ ) if $j>0$ and as outgoing road (i.e., at point $a_{i}$ ) if $j<0$. For each junction $J \in 2$, we indicate by $\operatorname{Inc}(J)$ the set of incoming roads, that are $I_{i}$ 's such that $i \in J$, while by $\operatorname{Out}(J)$ the set of outgoing roads, that are $I_{i}$ 's such that $-i \in J$. We assume that each road is incoming for (at most) one node and outgoing for (at most) one node.

In what follows, we suppose that $f_{k}=f$ for $k=1, \ldots, N$, but it is possible to generalize all definitions and results to the case of different fluxes $f_{k}$ for each road $I_{k}$. In fact, all statements are in terms of fluxes values at junctions, thus it is sufficient that the ranges of fluxes intersect. On each road, we consider the LWR model, described by the equation (see $[10,11])$,

$$
\rho_{t}+f(\rho)_{x}=0,
$$

where $(t, x) \in \mathbb{R}^{+} \times \mathbb{R}, \rho=\rho(t, x) \in\left[0, \rho_{\max }\right]$ is the density of cars, $\rho_{\max }$ is the maximal density, $f(\rho)=\rho v$ is the flux, and $v=v(\rho)$ the average velocity. For roads such that $i \notin \bigcup_{J \in 2} \operatorname{Inc}(J)$ and $b_{i}<+\infty$ or such that $i \notin \bigcup_{J \in 2} \operatorname{Out}(J)$ and $a_{i}>-\infty$, a boundary condition is needed. In throughout the paper, we use the following flux function:

$$
f(\rho)=\rho(1-\rho), \quad \rho \in[0,1]
$$

with a unique maximum $\sigma=1 / 2$.

For a single conservation law (2.1) on a real line $\mathbb{R}$, a Riemann Problem (RP), the basic ingredient to construct approximate solutions to Cauchy Problems with wave front tracking algorithm, is a Cauchy problem for an initial data piecewise constant with only one discontinuity. In a similar way, an RP at a junction is a Cauchy Problem for an initial data constant on each incoming and outgoing road.

Fix a junction $J$ with $n$ incoming roads and $m$ outgoing roads (junction of $n \times m$ type), where $I_{i}, i=1, \ldots, n$, are the incoming roads and $I_{j}, j=n+1, \ldots, n+m$, are the outgoing ones. Let $\rho=\left(\rho_{1}, \ldots, \rho_{n+m}\right), \rho_{k} \in[0,+\infty] \times I_{k}$ be the density vector for $J$.

Definition 2.2. A Riemann Solver (RS) for the junction $J$ is a map RS : $[0,1]^{n} \times[0,1]^{m} \mapsto$ $[0,1]^{n} \times[0,1]^{m}$ that associates to a Riemann datum $\rho_{0}=\left(\rho_{1,0}, \ldots, \rho_{n+m, 0}\right)$ at $J$ a vector $\hat{\rho}=$ $\left(\widehat{\rho}_{1}, \ldots, \hat{\rho}_{n+m}\right)$ so that the solution on $I_{i}, i=1, \ldots, n$, is given by the wave $\left(\rho_{i, 0}, \hat{\rho}_{i}\right)$ and on $I_{j}, j=n+1, \ldots, n+m$ is given by the wave $\left(\widehat{\rho}_{j}, \rho_{j, 0}\right)$. We require the consistency condition:

$$
\text { (CC) } \operatorname{RS}\left(\operatorname{RS}\left(\rho_{0}\right)\right)=\operatorname{RS}\left(\rho_{0}\right) \text {. }
$$

If $m \geq n$, it is possible to introduce an RS, based on the following rules $([1,12])$.

(A) At each junction $J$, we define a matrix $A=\left(\alpha_{j, i}\right)$, that describes the traffic distribution from incoming to outgoing roads, where, for every $i \in\{1, \ldots, n\}$ and $j \in\{n+1, \ldots, n+m\}, 0 \leq \alpha_{j, i} \leq 1$ and $\sum_{j=n+1}^{n+m} \alpha_{j, i}=1$. The $i$ th column of $A$ indicates 
the percentages of traffic that, from the incoming road $I_{i}$, are distributed to outgoing roads.

(B) respecting (A), drivers behave so as to maximize the flux through $J$.

In the case $m<n$, with $n \geq 2$ and $m=1$, in order to define an RS, we fix also the right of way parameters vector $p=\left(p_{1}, \ldots, p_{n}\right)$ with $\sum_{i=1}^{n} p_{i}=1$ and consider the following additional rule.

(P) Assume that not all cars can enter the outgoing roads, and let $C$ be the amount that can do it. Then $p_{i} C, i=1, \ldots, n$ cars come from the road $\mathrm{I}_{i}$ into the node.

For simplicity, we indicate by $\rho_{i}(t, x), i=1, \ldots, n$, the densities of the cars on the incoming roads and by $\rho_{j}(t, x), j=n+1, \ldots, n+m$, those on outgoing roads. Let us introduce the notation

$$
\gamma_{k}=f\left(\rho_{k}\right), \quad \widehat{\gamma}_{k}=f\left(\hat{\rho}_{k}\right), \quad k=1, \ldots, n+m .
$$

Proposition 2.3. Let $\left(\rho_{1,0}, \ldots, \rho_{n+m, 0}\right) \in[0,1]$ be the initial densities of an $R P$ at $J$. The maximum fluxes that can be obtained on the incoming roads and the outgoing ones, respectively, are given by (for a proof see [12]):

$$
\begin{gathered}
r_{i}^{\max }=\left\{\begin{array}{c}
f\left(\rho_{i, 0}\right), \quad \text { if } \rho_{i, 0} \in\left[0, \frac{1}{2}\right] \\
f\left(\frac{1}{2}\right)=\frac{1}{4} \text { if } \rho_{i, 0} \in\left[\frac{1}{2}, 1\right], \quad i=1, \ldots, n,
\end{array}\right. \\
r_{j}^{\max }=\left\{\begin{array}{c}
f\left(\frac{1}{2}\right)=\frac{1}{4}, \quad \text { if } \rho_{j, 0} \in\left[0, \frac{1}{2}\right] \\
\left.\left.f\left(\rho_{j, 0}\right) \quad \text { if } \rho_{j, 0} \in\right] \frac{1}{2}, 1\right], \quad j=n+1, \ldots, n+m .
\end{array}\right.
\end{gathered}
$$

Now we focus on junctions of $2 \times 1$ type and indicate with 1 and 2 the entering roads and with 3 the exiting one. In this case, we need only one right of way parameter $p$. The solution to the RP with initial data $\left(\rho_{1,0}, \rho_{2,0}, \rho_{3,0}\right)$ is constructed in the following way. Since we want to maximize the through traffic (rule (B)), we set

$$
\widehat{\gamma}_{3}=\min \left\{r_{1}^{\max }+r_{2}^{\max }, r_{3}^{\max }\right\}
$$

If $\widehat{\gamma}_{3}=r_{1}^{\max }+r_{2}^{\max }$, then the solution of the RP is $\widehat{\gamma}=\left(r_{1}^{\max }, r_{2}^{\max }, \gamma_{1}^{\max }+r_{2}^{\max }\right)$. Consider now the case $\widehat{\gamma}_{3}=r_{3}^{\max }$ and the following conditions:
(A1) $p r_{3}^{\max }<r_{1}^{\max }$
(A2) $(1-p) \gamma_{3}^{\max }<\gamma_{2}^{\max }$.

The solutions of the RP are the following:

(i) $\left(p \gamma_{3}^{\max },(1-p) \gamma_{3}^{\max }, \gamma_{3}^{\max }\right)$ if $A_{1}$ and $A_{2}$ are both satisfied;

(ii) $\left(\gamma_{3}^{\max }-\gamma_{2}^{\max }, \gamma_{2}^{\max }, \gamma_{3}^{\max }\right)$ if $A_{1}$ is satisfied and $A_{2}$ is not satisfied;

(iii) $\left(r_{1}^{\max }, r_{3}^{\max }-r_{1}^{\max }, r_{3}^{\max }\right)$ if $A_{2}$ is satisfied and $A_{1}$ is not satisfied. 
The case of both $A_{1}$ and $A_{2}$ false is not possible, since it would be $\gamma_{3}^{\max }>\gamma_{1}^{\max }+\gamma_{2}^{\max }$.

From the flux function, we can express $\widehat{\rho}_{k}$ in terms of $\widehat{\gamma}_{k}$. In fact, solving the equation $\widehat{\rho}_{k}\left(1-\widehat{\rho}_{k}\right)=\widehat{\gamma}_{k}$, we get

$$
\widehat{\rho}_{k}=\frac{1+s_{k} \sqrt{1-4 \widehat{\gamma}_{k}}}{2}, \quad k=1,2,3
$$

with

$$
\begin{aligned}
& s_{i}= \begin{cases}-1 \quad & \text { if } \rho_{i, 0}<\sigma, \gamma_{1}^{\max }+\gamma_{2}^{\max } \leq \gamma_{3}^{\max }, \\
& \text { or } \rho_{i, 0}<\sigma, \gamma_{3}^{\max }<\gamma_{1}^{\max }+\gamma_{2}^{\max }, p_{i} \widehat{\gamma}_{3} \geq \gamma_{i}^{\max }, \quad i=1,2, \\
+1 \quad & \text { if } \rho_{i, 0} \geq \sigma, \\
& \quad \text { or } \rho_{i, 0}<\sigma, \gamma_{3}^{\max }<\gamma_{1}^{\max }+\gamma_{2}^{\max }, p_{i} \widehat{\gamma}_{3}<\gamma_{i}^{\max },\end{cases} \\
& s_{3}= \begin{cases}-1 & \text { if } \rho_{3,0} \leq \sigma, \\
& \text { or } \rho_{3,0}>\sigma, \gamma_{1}^{\max }+\gamma_{2}^{\max }<\gamma_{3}^{\max }, \\
+1 & \text { if } \rho_{3,0}>\sigma, \gamma_{1}^{\max }+\gamma_{2}^{\max } \geq \gamma_{3}^{\max },\end{cases}
\end{aligned}
$$

where

$$
p_{i}=\left\{\begin{array}{cc}
p & \text { if } i=1 \\
1-p & \text { if } i=2
\end{array}\right.
$$

The velocity, in terms of $\widehat{\gamma}_{k}$, is given by $v\left(\widehat{\rho}_{k}\right)=\left(1-s_{k} \sqrt{1-4 \widehat{\gamma}_{k}}\right) / 2, k=1,2,3$.

\section{Cost Functionals}

In this section, we introduce the functionals used to evaluate the network performance and report optimization results for the functionals $J_{6}$ and $J_{7}$, compared with $J_{1}$ and $J_{2}$. We focus again on junctions of $2 \times 1$ type and we define the following functionals:

$J_{1}$ measuring car average velocity:

$$
J_{1}(t)=\sum_{k=1}^{3} \int_{I_{k}} v\left(\rho_{k}(t, x)\right) d x
$$


$J_{2}$ measuring average traveling time:

$$
J_{2}(t)=\sum_{k=1}^{3} \int_{I_{k}} \frac{1}{v\left(\rho_{k}(t, x)\right)} d x
$$

$J_{3}$ measuring total flux of cars:

$$
J_{3}(t)=\sum_{k=1}^{3} \int_{I_{k}} f\left(\rho_{k}(t, x)\right) d x
$$

$J_{4}$ measuring car density:

$$
J_{4}(t)=\sum_{k=1}^{3} \int_{0}^{t} \int_{I_{k}} \rho_{k}(\tau, x) d \tau d x,
$$

$J_{5}$ the Stop and Go Waves functional, measuring the velocity variation:

$$
J_{5}(t)=\mathrm{SGW}=\sum_{k=1}^{3} \int_{0}^{t} \int_{I_{k}}|D v(\rho)| d \tau d x,
$$

where $|D v|$ is the total variation of the distributional derivative $D \rho$, which is a finite Radon measure,

$J_{6}$ measuring the kinetic energy:

$$
J_{6}(t)=\sum_{k=1}^{3} \int_{I_{k}} f\left(\rho_{k}(t, x)\right) v\left(\rho_{k}(t, x)\right) d x
$$

$J_{7}$ measuring the average traveling time weighted with the number of cars moving on each road $I_{k}$ :

$$
J_{7}(t)=\sum_{k=1}^{3} \int_{I_{k}} \frac{\rho_{k}(t, x)}{v\left(\rho_{k}(t, x)\right)} d x
$$

For a fixed time horizon $[0, T]$, our aim is to maximize $\int_{0}^{T} J_{1}(t) d t, \int_{0}^{T} J_{3}(t) d t, \int_{0}^{T} J_{6}(t) d t$ and to minimize $\int_{0}^{T} J_{2}(t) d t, \int_{0}^{T} J_{7}(t) d t$, choosing the right of way parameter $p_{k}(t)$. Since the solutions of such optimization control problems are too difficult, we reduce to the following problem. 
$\left(P_{r}\right)$ Consider a junction $J$ of $2 \times 1$ type, the functionals $J_{k}, k=1,2,3,6,7$, and the right of way parameter $p_{k}$ as controls. We want to minimize $J_{2}(T), J_{7}(T)$ and to maximize $J_{1}(T), J_{3}(T), J_{6}(T)$ for $T$ sufficiently big.

Given the initial data, solving the RP, we determine the average velocity, the average traveling time, and the flux over the network as function of the right of way parameters $p$, then all the functionals depend on $p$. As was proved in [6-8], the functional $J_{3}(T)$ does not depend on the right of way parameter.

\subsection{Optimization of $J_{6}$ and $J_{7}$}

Let us consider the optimization of the functionals, measuring kinetic energy and weighted average traveling time. For $T$ sufficiently big the functionals assume the form:

$$
\begin{aligned}
& J_{6}(T)=\sum_{k=1}^{3} f\left(\widehat{\rho}_{k}\right) v\left(\widehat{\rho}_{k}\right)=\sum_{k=1}^{3} \widehat{\gamma}_{k} \frac{\left(1-s_{k} \sqrt{1-4 \widehat{\gamma}_{k}}\right)}{2}, \\
& J_{7}(T)=\sum_{k=1}^{3} \frac{\hat{\rho}_{k}}{v\left(\hat{\rho}_{k}\right)}=\sum_{i=1}^{3} \frac{1+s_{k} \sqrt{1-4 \widehat{\gamma}_{k}}}{1-s_{k} \sqrt{1-4 \widehat{\gamma}_{k}}}
\end{aligned}
$$

where $s_{k}, k=1,2,3$ are defined in (2.8), $\hat{\rho}=\left(\widehat{\rho}_{1}, \hat{\rho}_{2}, \widehat{\rho}_{3}\right)=\operatorname{RS}\left(\rho_{1,0}, \rho_{2,0}, \rho_{3,0}\right)$, and $\hat{\gamma}=f(\widehat{\rho})$.

If $\widehat{\gamma}_{3}=\gamma_{1}^{\max }+\gamma_{2}^{\max }$, the solution of the RP does not depend on the parameter $p$ and the same happens for the functionals $J_{6}$ and $J_{7}$ which do not depend on the parameter $p$. Hence we analyze the functionals in the case $\gamma_{3}^{\max }<\gamma_{1}^{\max }+\gamma_{2}^{\max }$, that is, $\widehat{\gamma}_{3}=\gamma_{3}^{\max }$. Let us define

$$
\begin{gathered}
\beta^{-}=\frac{r_{3}^{\max }-r_{1}^{\max }}{r_{1}^{\max }}, \quad p^{+}=\frac{1}{1+\beta^{-}}=\frac{r_{1}^{\max }}{r_{3}^{\max }}, \\
\beta^{+}=\frac{r_{2}^{\max }}{r_{3}^{\max }-r_{2}^{\max }}, \quad p^{-}=\frac{1}{1+\beta^{+}}=\frac{r_{3}^{\max }-r_{2}^{\max }}{r_{3}^{\max }} .
\end{gathered}
$$

It is easy to check that $\beta^{-} \leq \beta^{+}$. Then, for $p \geq p^{+}, A_{1}$ is false and $A_{2}$ is true, for $p \leq p^{-}, A_{1}$ is true and $A_{2}$ is false and, finally, for $p^{-}<p<p^{+}, A_{1}$ and $A_{2}$ are both true. From (2.7), neglecting the parts of the costs that do not depend on $p$, maximizing $J_{6}$ and minimizing $J_{7}$ is equivalent to maximize and minimize, respectively,

$$
\tilde{J}_{6}= \begin{cases}\left(\gamma_{3}^{\max }-r_{2}^{\max }\right)\left(1-s_{1} \sqrt{1-4\left(\gamma_{3}^{\max }-r_{2}^{\max }\right)}\right)+r_{2}^{\max }\left(1-s_{2} \sqrt{1-4 \gamma_{2}^{\max }}\right), & 0 \leq p \leq p^{-}, \\ p \gamma_{3}^{\max }\left(1-s_{1} \sqrt{1-4 p \gamma_{3}^{\max }}\right)+(1-p) \gamma_{3}^{\max }\left(1-s_{2} \sqrt{1-4(1-p) r_{3}^{\max }}\right), & p^{-}<p<p^{+}, \\ r_{1}^{\max }\left(1-s_{1} \sqrt{1-4 \gamma_{1}^{\max }}\right)+\left(r_{3}^{\max }-r_{1}^{\max }\right)\left(1-s_{2} \sqrt{1-4\left(r_{3}^{\max }-\gamma_{1}^{\max }\right)}\right), & p^{+} \leq p \leq 1,\end{cases}
$$




$$
\tilde{J}_{7}= \begin{cases}\frac{1+s_{1} \sqrt{1-4\left(\gamma_{3}^{\max }-\gamma_{2}^{\max }\right)}}{1-s_{1} \sqrt{1-4\left(\gamma_{3}^{\max }-\gamma_{2}^{\max }\right)}}+\frac{1+s_{2} \sqrt{1-4 \gamma_{2}^{\max }}}{1-s_{2} \sqrt{1-4 \gamma_{2}^{\max }}}, \quad 0 \leq p \leq p^{-}, \\ \frac{1+s_{1} \sqrt{1-4 p r_{3}^{\max }}}{1-s_{1} \sqrt{1-4 p r_{3}^{\max }}}+\frac{1+s_{2} \sqrt{1-4(1-p) r_{3}^{\max }}}{1-s_{2} \sqrt{1-4(1-p) r_{3}^{\max }}}, \quad p^{-}<p<p^{+}, \\ \frac{1+s_{1} \sqrt{1-4 \gamma_{1}^{\max }}}{1-s_{1} \sqrt{1-4 \gamma_{1}^{\max }}}+\frac{1+s_{2} \sqrt{1-4\left(r_{3}^{\max }-r_{1}^{\max }\right)}}{1-s_{2} \sqrt{1-4\left(r_{3}^{\max }-\gamma_{1}^{\max }\right)},}, \quad p^{+} \leq p \leq 1 .\end{cases}
$$

Observe that when the condition $A_{1}$ is false and $A_{2}$ is true, or vice versa, the cost functionals $J_{6}$ and $J_{7}$ are constant with respect to $p$. To complete the analysis of the costs it is enough to take the derivatives with respect to $p$ in the region where both $A_{1}$ and $A_{2}$ are true. The expressions are a bit long, so we do not report them, but it is straightforward to check that $J_{6}$ and $J_{7}$ are decreasing for $p<1 / 2$ and increasing for $p>1 / 2$, hence they have a minimum in $p=1 / 2$.

We analyze the functionals $J_{6}$ and $J_{7}$ in the intervals: $\left.\left[0, p^{-}\right],\right] p^{-}, p^{+}\left[\right.$and $\left[p^{+}, 1\right]$. We search for absolute minimum in the case of $J_{6}$ and absolute maximum in the analysis of $J_{7}$, taking into account the possible values that $s_{k}, k=1,2,3$, can assume in the above intervals:

(i) $s_{1}=s_{2}=1, s_{3}= \pm 1$ in $\left[0, p^{-}\right], s_{1}=s_{2}=1, s_{3}= \pm 1$ in $] p^{-}, p^{+}\left[, s_{1}=s_{2}=1, s_{3}= \pm 1\right.$ in $\left[p^{+}, 1\right]$;

(ii) $s_{1}=s_{2}=1, s_{3}= \pm 1$ in $\left[0, p^{-}\right], s_{1}=s_{2}=1, s_{3}= \pm 1$ in $] p^{-}, p^{+}\left[, s_{1}=-1, s_{2}=1, s_{3}= \pm 1\right.$ in $\left[p^{+}, 1\right]$;

(iii) $s_{1}=1, s_{2}=-1, s_{3}= \pm 1$ in $\left[0, p^{-}\right], s_{1}=s_{2}=1, s_{3}= \pm 1$ in $] p^{-}, p^{+}\left[, s_{1}=s_{2}=1, s_{3}= \pm 1\right.$ in $\left[p^{+}, 1\right]$;

(iv) $s_{1}=1, s_{2}=-1, s_{3}= \pm 1$ in $\left[0, p^{-}\right], s_{1}=s_{2}=1, s_{3}= \pm 1$ in $] p^{-}, p^{+}\left[, s_{1}=-1, s_{2}=1, s_{3}=\right.$ \pm 1 in $\left[p^{+}, 1\right]$.

Remark 3.1. Since $s_{3}$ assumes the same values, 1 or -1 , in all the intervals, (hence in the point of discontinuities) and the terms in which $s_{3}$ occurs do not depend on $p$, the optimal values are independent from $s_{3}$.

The functionals $J_{2}(T)$ and $J_{7}(T)$ are maximized for the same values of $p$. In fact we get the following theorem.

Theorem 3.2. Consider a junction $J$ of $2 \times 1$ type. For the flux function (2.2), and $T$ sufficiently big, the cost functionals $J_{2}(T)$ and $J_{7}(T)$ are optimized for the following values of $p$.

(1) Case $s_{1}=s_{2}=+1$, we have that

(a) $p=1 / 2$ if $\beta^{-} \leq 1 \leq \beta^{+}$or $\gamma_{2}^{\max }=\gamma_{3}^{\max }$;

(b) $p \in\left[0, p^{-}\right]$if $\beta^{-} \leq \beta^{+} \leq 1$;

(c) $p \in\left[p^{+}, 1\right]$ if $1 \leq \beta^{-} \leq \beta^{+}$; 
(2) Case $s_{1}=-1=-s_{2}$, we have that

(a) $p=1 / 2$ or $p \in\left[p^{+}, 1\right]$ if $\beta^{-} \leq 1 \leq \beta^{+}$or $\gamma_{2}^{\max }=\gamma_{3}^{\max }$;

(b) $p \in\left[0, p^{-}\right]$or $p \in\left[p^{+}, 1\right]$ if $\beta^{-} \leq \beta^{+} \leq 1$;

(c) $p \in\left[p^{+}, 1\right]$ if $1 \leq \beta^{-} \leq \beta^{+}$;

(3) Case $s_{1}=+1=-s_{2}$, we have that

(a) $p=1 / 2$ or $p \in\left[0, p^{-}\right]$if $\beta^{-} \leq 1 \leq \beta^{+}$;

(b) $p \in\left[0, p^{-}\right]$if $\beta^{-} \leq \beta^{+} \leq 1$;

(c) $p \in\left[0, p^{-}\right]$or $p \in\left[p^{+}, 1\right]$ if $1 \leq \beta^{-} \leq \beta^{+}$;

(d) $p=1 / 2$ or $p \in\left[p^{+}, 1\right]$ if $\gamma_{2}^{\max }=\gamma_{3}^{\max }$;

(4) Case $s_{1}=s_{2}=-1$, we have that

(a) $p=1 / 2$ or $p \in\left[p^{+}, 1\right]$ if $\beta^{-} \leq 1 \leq \beta^{+}$, with $\beta^{-} \beta^{+}>1$ or $\gamma_{2}^{\max }=\gamma_{3}^{\max }$;

(b) $p=1 / 2$ or $p \in\left[0, p^{-}\right]$if $\beta^{-} \leq 1 \leq \beta^{+}$, with $\beta^{-} \beta^{+}<1$;

(c) $p=1 / 2$ or $p \in\left[0, p^{-}\right] \cup\left[p^{+}, 1\right]$ if $\beta^{-} \leq 1 \leq \beta^{+}$, with $\beta^{-} \beta^{+}=1$;

(d) $p \in\left[0, p^{-}\right]$if $\beta^{-} \leq \beta^{+} \leq 1$,

(e) $p \in\left[p^{+}, 1\right]$, if $1 \leq \beta^{-} \leq \beta^{+}$;

where $\beta^{-}=\left(\gamma_{3}^{\max }-\gamma_{1}^{\max }\right) / \gamma_{1}^{\max }, \beta^{+}=\gamma_{2}^{\max } /\left(\gamma_{3}^{\max }-\gamma_{2}^{\max }\right), p^{+}=\gamma_{1}^{\max } / \gamma_{3}^{\max }, p^{-}=\left(\gamma_{3}^{\max }-\right.$ $\left.\gamma_{2}^{\max }\right) / \gamma_{3}^{\max }$. $p=1 / 2$.

In the particular case $r_{1}^{\max }=\gamma_{2}^{\max }=\gamma_{3}^{\max }$, the functionals $J_{2}$ and $J_{7}$ are optimized for

The maximization of the functionals $J_{1}(T)$ and $J_{6}(T)$ is reached, in some cases, for different values of the right of way parameter, as reported in the following theorem, in which the optimization analysis of the new functional $J_{6}(T)$ is compared with the results obtained in [6] for $J_{1}(T)$.

Theorem 3.3. Consider a junction $J$ of $2 \times 1$ type. For the flux function (2.2), and $T$ sufficiently big, the cost functionals $J_{1}(T)$ and $J_{6}(T)$ are optimized for the following values of $p$.

(1) Case $s_{1}=s_{2}=+1$, we have that

(a) $p \in\left[0, p^{-}\right]$if $\beta^{-} \leq 1 \leq \beta^{+}$, with $\beta^{-} \beta^{+}>1$, or $1 \leq \beta^{-} \leq \beta^{+}$, or $\gamma_{2}^{\max }=\gamma_{3}^{\max }$;

(b) $p \in\left[0, p^{-}\right] \cup\left[p^{+}, 1\right]$ if $\beta^{-} \leq 1 \leq \beta^{+}$, with $\beta^{-} \beta^{+}=1$;

(c) $p \in\left[p^{+}, 1\right]$ if $\beta^{-} \leq 1 \leq \beta^{+}$, with $\beta^{-} \beta^{+}<1$ or $\beta^{-} \leq \beta^{+} \leq 1$;

(2) Case $s_{1}=-1=-s_{2}$, we have that

for $J_{1}(T), p \in\left[p^{+}, 1\right]$;

for $J_{6}(T)$,

(a) $p \in\left[p^{+}, 1\right]$ if $\beta^{-} \leq 1 \leq \beta^{+}$, or $\beta^{-} \leq \beta^{+} \leq 1$;

(b) $p \in\left[0, p^{-}\right]$or $p \in\left[p^{+}, 1\right]$ if $1 \leq \beta^{-} \leq \beta^{+}$, or $\gamma_{2}^{\max }=\gamma_{3}^{\max }$;

(3) Case $s_{1}=+1=-s_{2}$, we have that 
for $J_{1}(T), p \in\left[0, p^{-}\right]$;

for $J_{6}(T)$,

(a) $p \in\left[0, p^{-}\right]$if $\beta^{-} \leq 1 \leq \beta^{+}$, or $1 \leq \beta^{-} \leq \beta^{+}$, or $\gamma_{2}^{\max }=\gamma_{3}^{\max }$;

(b) $p \in\left[0, p^{-}\right]$or $p \in\left[p^{+}, 1\right]$ if $\beta^{-} \leq \beta^{+} \leq 1$;

(4) Case $s_{1}=s_{2}=-1$, we have that

for $J_{1}(T)$,

(a) $p \in\left[0, p^{-}\right]$if $\beta^{-} \leq 1 \leq \beta^{+}$, with $\beta^{-} \beta^{+}<1$, or $\beta^{-} \leq \beta^{+} \leq 1$;

(b) $p \in\left[0, p^{-}\right] \cup\left[p^{+}, 1\right]$ if $\beta^{-} \leq 1 \leq \beta^{+}$, with $\beta^{-} \beta^{+}=1$;

(c) $p \in\left[p^{+}, 1\right]$ if $\beta^{-} \leq 1 \leq \beta^{+}$, with $\beta^{-} \beta^{+}>1$, or $1 \leq \beta^{-} \leq \beta^{+}$, or $\gamma_{2}^{\max }=\gamma_{3}^{\max }$;

for $J_{6}(T)$,

(a) $p \in\left[0, p^{-}\right]$or $p \in\left[p^{+}, 1\right]$ if $\beta^{-} \leq 1 \leq \beta^{+}$, with $\beta^{-} \beta^{+}>1$, or $\beta^{-} \beta^{+}<1$, or $\gamma_{2}^{\max }=\gamma_{3}^{\max }$;

(b) $p \in\left[0, p^{-}\right] \cup\left[p^{+}, 1\right]$ if $\beta^{-} \leq 1 \leq \beta^{+}$, with $\beta^{-} \beta^{+}=1$;

(c) $p \in\left[p^{+}, 1\right]$ if $\beta^{-} \leq \beta^{+} \leq 1$;

(d) $p \in\left[0, p^{-}\right]$if $1 \leq \beta^{-} \leq \beta^{+}$;

where $\beta^{-}=\left(\gamma_{3}^{\max }-\gamma_{1}^{\max }\right) / \gamma_{1}^{\max }, \beta^{+}=\gamma_{2}^{\max } /\left(\gamma_{3}^{\max }-\gamma_{2}^{\max }\right), p^{+}=\gamma_{1}^{\max } / \gamma_{3}^{\max }, p^{-}=\left(\gamma_{3}^{\max }-\right.$ $\left.\gamma_{2}^{\max }\right) / \gamma_{3}^{\max }$.

The functionals $J_{1}$ and $J_{6}$ are maximized for $p=0$ or $p=1$ if $\gamma_{1}^{\max }=\gamma_{2}^{\max }=\gamma_{3}^{\max }$.

Remark 3.4. In the cases in which two possible optimal candidates exist, we evaluate the corresponding values of the cost functionals. The optimal $p$ is given by the value that minimizes or maximizes, respectively, the functionals $J_{1}(T), J_{6}(T)$ and $J_{2}(T), J_{7}(T)$. If we have a set of optimal values $\left[0, p^{-}\right]$, we can choose $p=p^{-}$or $p=p^{-}-\varepsilon$, while in the case in which the optimal right of way parameter belongs to $\left[p^{+}, 1\right]$ we can take as optimal value $p=p^{+}$or $p=p^{+}+\varepsilon$, with $\varepsilon$ small and positive.

\section{Simulations}

In this section, we present some simulation results in order to test the optimization algorithms both for single junctions and complex networks. The aim is to verify the correctness of the analytical results and then to analyze the effects of different control procedures, applied locally at each junction, on the global performances of networks. The approximation of the conservation laws, that describe the density evolution for each road of the network (see [13]), is made by the numerical scheme of Godunov ([14]), with space step $\Delta x=0.01$. The time step is determined by the CFL condition ([15]), equal to 0.5 .

\subsection{Single Junctions}

We consider single junctions of $2 \times 1$ type, namely, junctions consisting of two incoming roads, 1 and 2, and one outgoing road, 3 , in order to verify the goodness of optimization procedures. Then we compare cost functionals behaviour using right of way parameters, that optimize the 


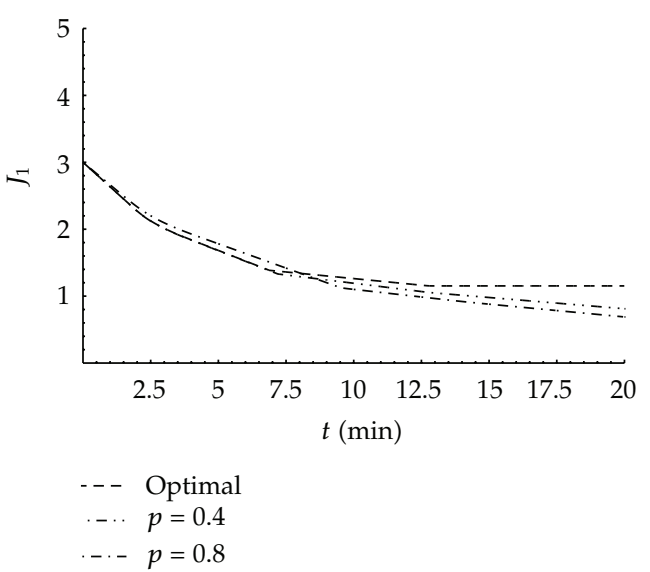

(a)

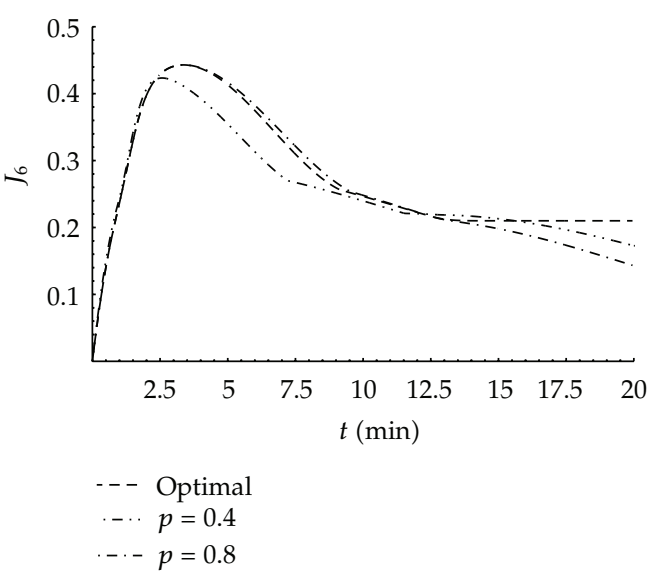

(b)

Figure 1: Comparison among $J_{1}$ computed by opt $J_{1}$ (a), $J_{6}$ computed by opt $J_{6}$ (b), and fixed cases for $p=0.4$ and $p=0.8$.

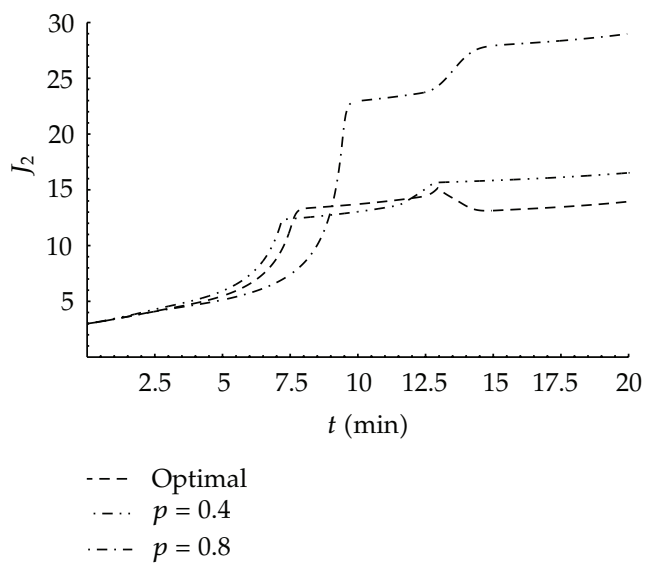

(a)

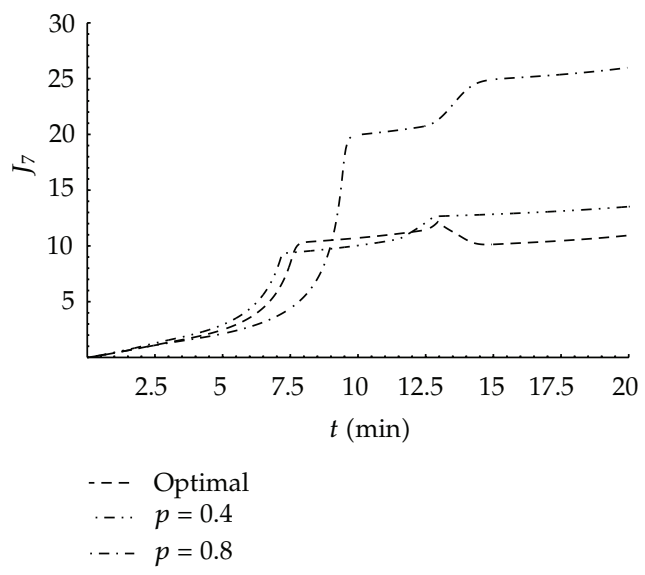

(b)

Figure 2: Comparison among $J_{2}(\mathrm{a})$ and $J_{7}(\mathrm{~b})$ computed by opt $J_{2} J_{7}$, and fixed cases for $p=0.4$ and $p=0.8$.

functionals (optimal case) and fixed right of way parameters ( fixed case), according to which $p$ is chosen by the user. The evolution of the traffic is simulated in a time interval $[0, T]$, where $T=20 \mathrm{~min}$ for the flux function (2.2). As for the initial conditions on the roads, we assume that, at the starting instant of simulation $(t=0)$, all roads are empty. Moreover, for roads 1 , 2 , and 3 , also boundary data, $\rho_{i, b}, i \in\{1,2,3\}$, have to be considered. Precisely, we choose $\rho_{i, b}=0.3, \rho_{2, b}=0.4$, and $\rho_{3, b}=0.1$.

The motivation for the choice of such boundary data is that it allows to capture the case in which different optimization procedures, one for $J_{1}$, indicated as "opt $J_{1}$ ", one for $J_{6}$, "opt $J_{6}$ ", and one for both $J_{2}$ and $J_{7}$, "opt $J_{2} J_{7}$ ", give origin to different optimal values.

As you can see from Figures 1 and 2, the optimal algorithms for $J_{1}, J_{2}, J_{6}$, and $J_{7}$ improve traffic conditions with respect to the fixed cases. Notice that, when we are not in the steady state, it could occur that some fixed simulations can behave better than the optimal 


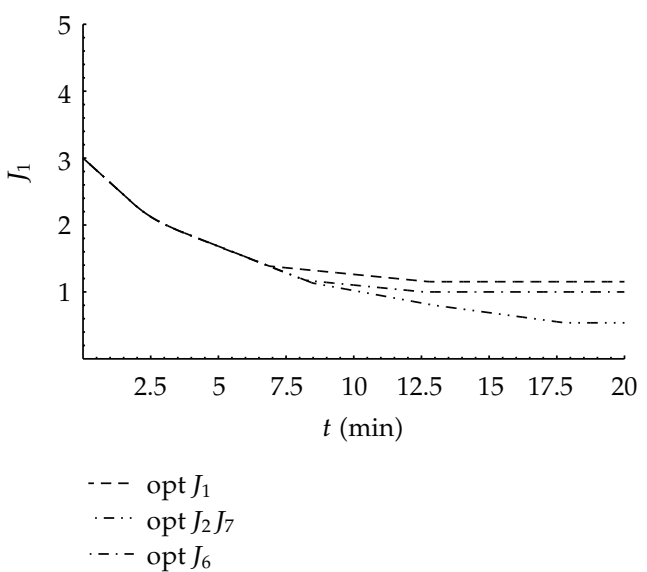

(a)

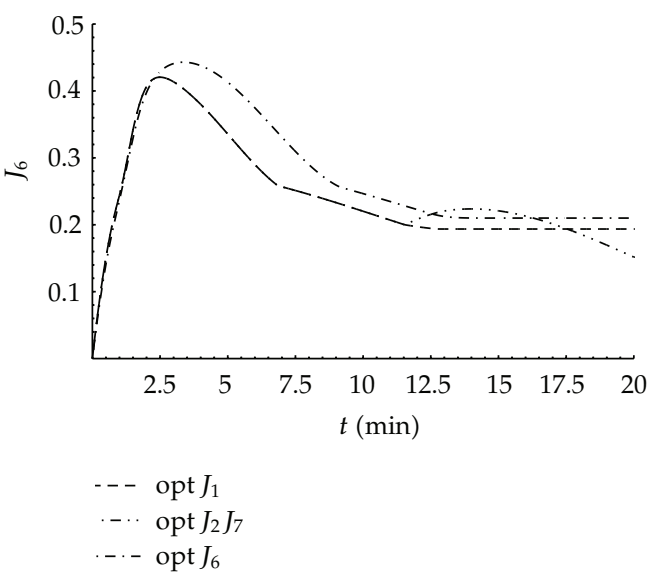

(b)

Figure 3: Comparison among $J_{1}(\mathrm{a})$ and $J_{6}$ evaluated by opt $J_{1}, \operatorname{opt} J_{2} J_{7}$ and $\operatorname{opt} J_{6}(\mathrm{~b})$.

Table 1: Boundary data for the four simulation cases.

\begin{tabular}{lccc}
\hline Case & $\rho_{1, b}$ & $\rho_{2, b}$ & $\rho_{3, b}$ \\
\hline$A$ & 0.112 & 0.139 & 0.846 \\
$B$ & 0.183 & 0.139 & 0.782 \\
$C$ & 0.112 & 0.183 & 0.673 \\
$D$ & 0.301 & 0.412 & 0.101 \\
\hline
\end{tabular}

ones. This is due to the fact that the analytical results are always obtained for big times. In Figure 3, we present the differences between the optimization algorithms for cost functionals $J_{1}$ and $J_{6}$. As expected, $J_{1}$ and $J_{6}$ are higher, respectively, applying opt $J_{1}$ and opt $J_{6}$. Moreover, simulating the junction with the parameter $p$ obtained using the control procedures opt $J_{1}$, opt $J_{6}$, and opt $J_{2} J_{7}$, the behaviours of $J_{1}$ and $J_{6}$ are different, confirming the theoretical results that, depending on the initial data, the functionals can be optimized for different values of the right of way parameter.

As for a single junction of $2 \times 1$ type, various optimization algorithms can be applied to set the priority parameters, we tried to understand which is the best policy to adopt for the improvements of traffic conditions. In order to discriminate among the different optimization approaches, we analyze the behaviour of the Stop and Go Waves functional (SGW), which measures the velocity variations on roads. We simulate the traffic evolution for a junction in four different situations, denoted by $A, B, C$, and $D$, in a time interval $[0, T]$, where $T$ is $30 \mathrm{~min}$ for cases $A$ and $B$ and $50 \mathrm{~min}$ for cases $C$ and $D$. We assume that at the beginning of the simulation all the roads are empty. Boundary data are reported in Table 1 , and chosen in such way to test various cases of Theorems 3.2 and 3.3. Table 2 .

For such case studies, optimal values for the right of way parameter $p$ are as shown in

In Figures 4 and 5, the behaviour of SGW, applying opt $J_{1}$, opt $J_{2} J_{7}$, and opt $J_{6}$, in the different simulation cases, is depicted. In the steady state, SGW is lower when the algorithm opt $J_{1}$ is used, and this implies a lower probability of car accidents. We can conclude that it is suitable to choose the parameter $p$ which maximizes the average velocities. Notice that in 
Table 2: Optimal right of way parameters for simulation cases $A, B, C$, and $D$.

\begin{tabular}{lccc}
\hline Case & opt $J_{1}$ & opt $J_{2} J_{7}$ & opt $J_{6}$ \\
\hline$A$ & $p \in[0.769,1]$ & $p=0.5$ & $p \in[0,0.769]$ \\
$B$ & $p \in[0,0.294]$ & $p \in[0,0.294]$ & $p \in[0.882,1]$ \\
$C$ & $p \in[0.454,1]$ & $p \in[0.454,1]$ & $p \in[0,0.318]$ \\
$D$ & $p \in[0,0.040]$ & $p=0.5$ & $p \in[0.840,1]$ \\
\hline
\end{tabular}

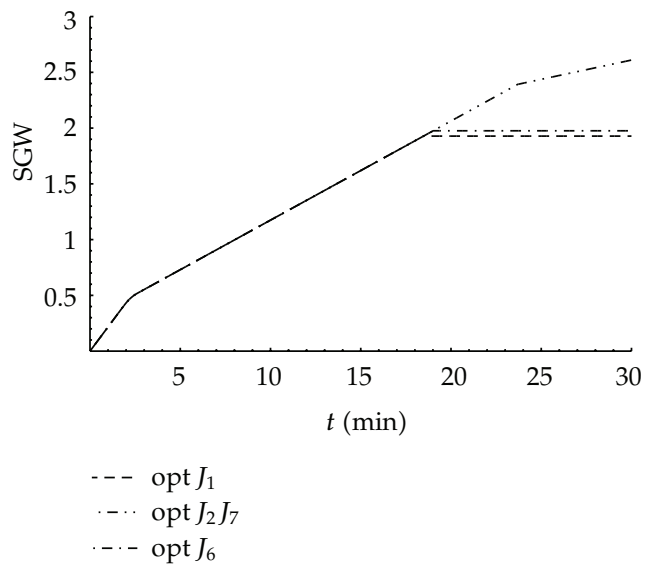

(a)

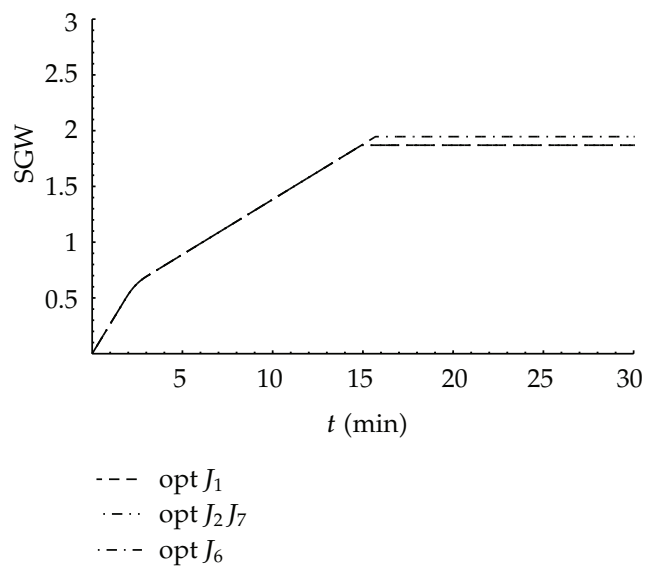

(b)

Figure 4: Comparison among SGW, evaluated by opt $J_{1}$, opt $J_{2} J_{7}$, and opt $J_{6}$, in case $A$ (a) and case $B$ (b).

cases $B$ and $C$, the optimal algorithms opt $J_{1}$ and opt $J_{2} J_{7}$ give the same set of $p$, hence curves overlap.

\subsection{Simulation of Traffic for Re Di Roma Square}

This subsection is devoted to the description of simulative results for a real urban network, Re di Roma Square, a big traffic circle inside the urban network of Rome, in Italy. The choice of this case study is justified by the presence of congestion phenomena, which could be avoided through an opportune choice of network parameters.

The topology of the square, represented in Figure 6, is described by 12 roads, which form the circle: $1 R, 2 R, 3 R, 4 R, 5 R, 6 R, 7 R, 8 R, 9 R, 10 R, 11 R$, and $12 R ; 12$ roads, connecting the inner roads with outside: aosta_ent, aosta_exi, vercelli, pinerolo, appia_sud_ent, appia_sud_exi, albalonga_ent, albalonga_exi, cerveteri_ent, cerveteri_exi, appia_nord_ent, appia_nord_exi.

As it is shown, Re di Roma square is formed by junctions of $2 \times 1$ type $(1,3,5,7,9,11)$, in white, and junctions of $1 \times 2$ type $(2,4,6,8,10,12)$, in black. The traffic distribution coefficients at $1 \times 2$ junctions are completely determined by road capacities (and the characteristics of the nearby portion of the Rome urban network); hence only right of way parameters for $2 \times 1$ junctions can be chosen as control parameters. Table 3 reports the distribution coefficients used for simulations.

The evolution of the traffic flows is simulated in a time interval $[0, T]$, where $T=$ $30 \mathrm{~min}$. We assume that, at the starting instant of simulation, all roads are empty. We use 


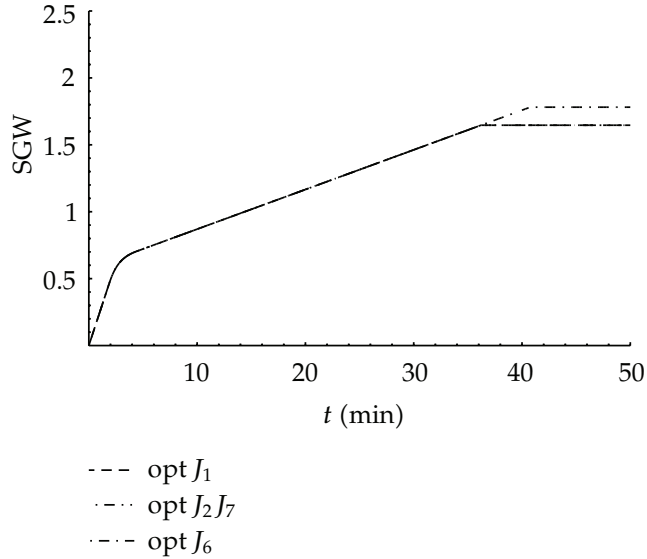

(a)

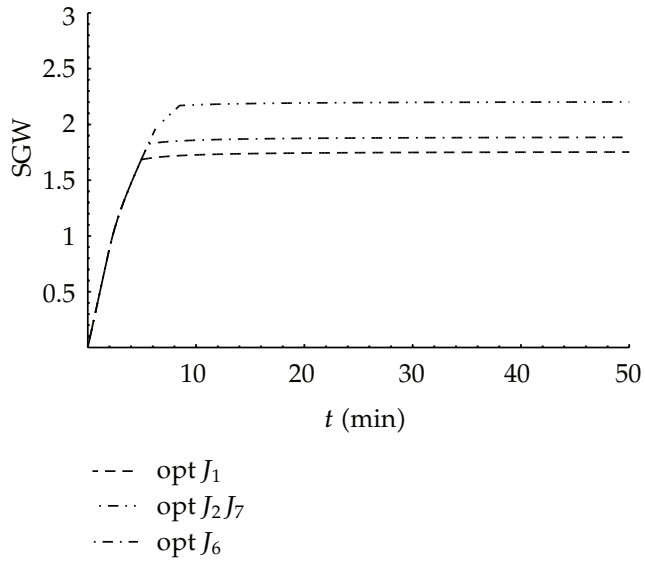

(b)

Figure 5: Comparison among SGW, evaluated by opt $J_{1}$, opt $J_{2} J_{7}$, and opt $J_{6}$, in case $C$ (a) and case $D$ (b).

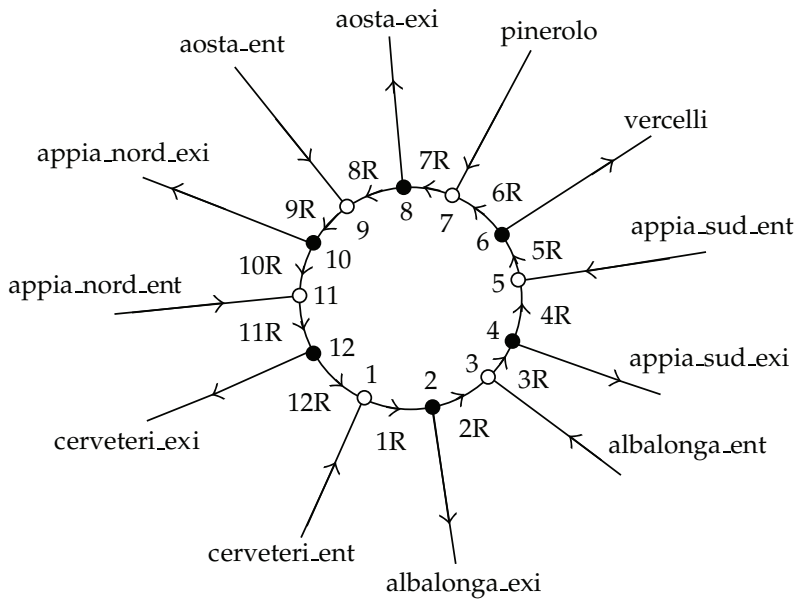

Figure 6: Topology of Re di Roma Square.

boundary conditions for roads with not infinite endpoints. In particular, roads cerveteri_exi, albalonga_exi, appia_sud_exi, vercelli, aosta_exi, and appia_nord_exi have a boundary data equal to 0.4 , while the other ones equal to 0.35 .

We analyze two simulation cases: right of way parameters, that optimize the cost functionals (optimal case); dynamic random parameters (dynamic random case), which means that right of way parameters change randomly at every step of the simulation process.

The aim is to investigate the effects of opt $J_{1}, \operatorname{opt} J_{6}$, and opt $J_{2} J_{7}$ on the global performances of the network in order to understand which is the best optimization algorithm (the algorithm realizing the better viability conditions on the whole Re di Roma square). In the following pictures, we report the time behaviour evolutions of the cost functionals $J_{1}, J_{2}$, $J_{6}$, and $J_{7}$, fixing at every junction the values of the right of way parameters obtained by the optimization procedures. 
Table 3: Traffic distribution parameters for junctions of $1 \times 2$ type.

\begin{tabular}{lcc}
\hline Junction $i$ & $\alpha_{i R,(i-1) R}$ & $\alpha_{i,(i-1) R}$ \\
\hline$i=2$ & 0.866071 & 0.133929 \\
$i=4$ & 0.459854 & 0.540146 \\
$i=6$ & 0.800971 & 0.199029 \\
$i=8$ & 0.730612 & 0.269388 \\
$i=10$ & 0.536050 & 0.463950 \\
$i=12$ & 0.753927 & 0.246073 \\
\hline
\end{tabular}

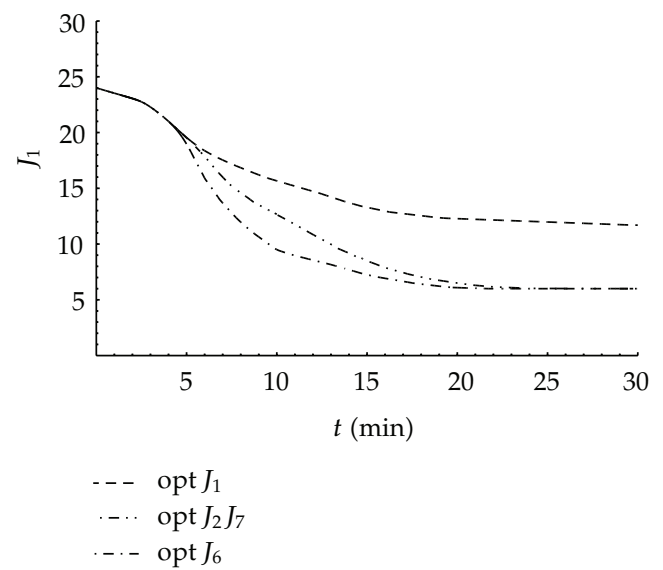

(a)

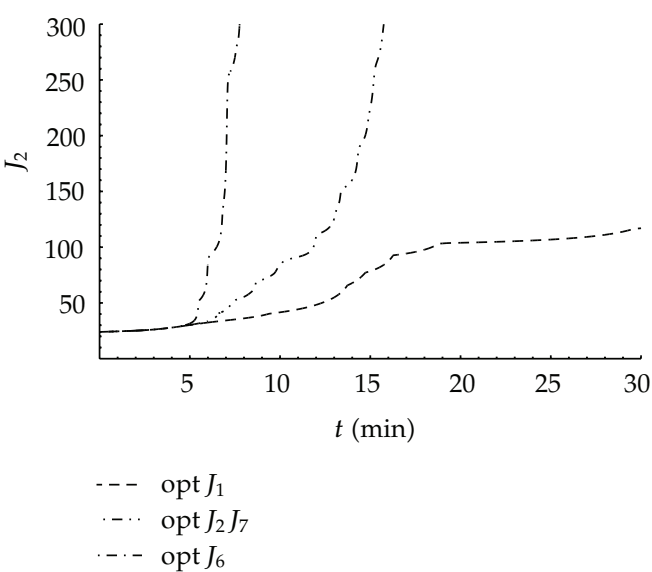

(b)

Figure 7: Behaviour of the optimal cost functionals $J_{1}(\mathrm{a})$ and $J_{2}(\mathrm{~b})$.

The optimizations of local type, like the ones that we are considering here, could not necessarily imply global optimization for big networks, as we can see in Figures 7, 8, 10, 11 . This is due to various factors, mainly depending on the network topology and on traffic loads.

First of all, notice that in the case study the algorithm opt $J_{1}$ allows a global optimization for the whole network. It is evident in Figure 7, where $J_{1}$ and $J_{2}$ are, respectively, the highest and the lowest. Hence, the use of opt $J_{2} J_{7}$ and opt $J_{6}$, as control procedures, does not guarantee better performance of traffic flows. The goodness of opt $J_{1}$ for global performances is confirmed by the behaviour of $J_{2}$. In fact, opt $J_{2} J_{7}$ and opt $J_{6}$ can let $J_{2}$ explode, that is, the traffic circle is stuck and the time to run inside goes to infinity. This situation is more evident in Figure 8, where we can capture another important aspect: the total kinetic energy $J_{6}$, on the whole network, tends to zero when opt $J_{1}$ is not used. This means that the cars flux is going to zero, as evident from Figure 9, hence roads inside the circle are becoming full. A consequence of this phenomenon is also visible in $J_{7}$ evolution, that tends to infinity.

In Figure 10, $J_{4}$ and $J_{5}$ (Stop and Go Waves functional, SGW) behaviour are depicted. There are no optimization algorithms for these functionals, and they are computed directly using opt $J_{1}$, opt $J_{2} J_{7}$, and opt $J_{6}$. It is evident that the amount of traffic load, visible in $J_{4}$, tends to decrease using opt $J_{1}$. Moreover, the behaviour of $J_{5}$, that measures the velocity variation, indicates that the use of opt $J_{1}$ leads to more regular densities on roads, giving advantages in terms of security. 


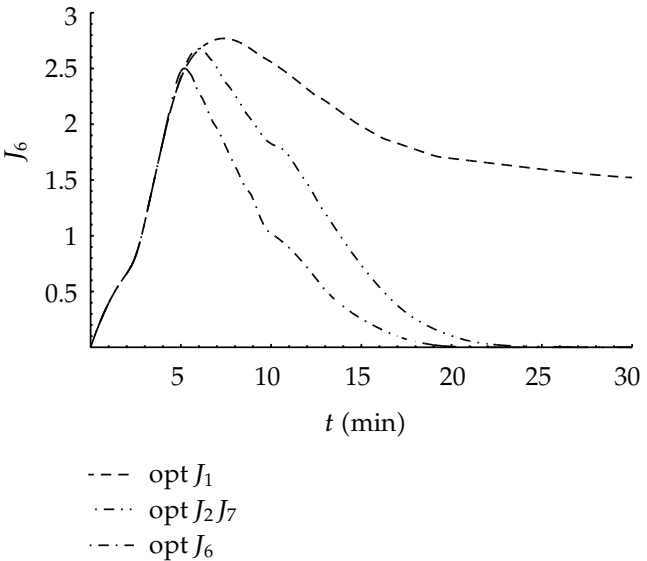

(a)

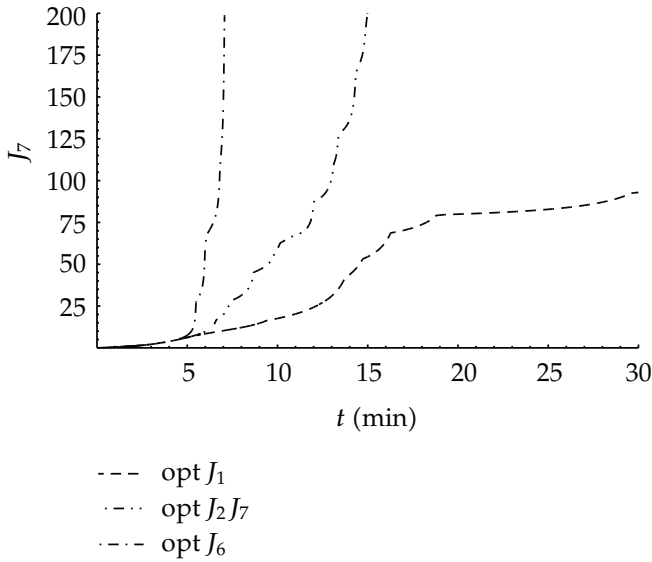

(b)

Figure 8: Behaviour of the optimal cost functionals $J_{6}(\mathrm{a})$ and $J_{7}(\mathrm{~b})$.

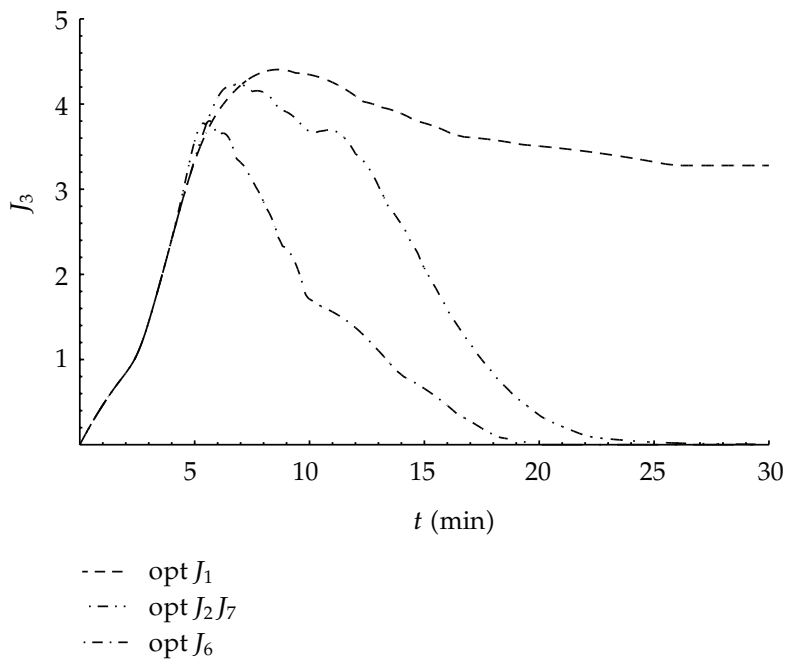

Figure 9: Behaviour of the cost functional $J_{3}$.

To complete the discussion, we focus the attention on the characteristics of dynamic random simulations (Figure 11). As proved in [8], the dynamic random simulation is similar to a fixed simulation with all right of way parameters equal to 0.5 . In fact, dynamic random choices fit well the optimizations obtained with $J_{2}$ and $J_{7}$, since in the considered case the optimal value is 0.5 for each junction of $2 \times 1$ type. Unlike the case presented in [6,8], opt $J_{1}$ does not guarantee an average optimal right of way parameter equal to 0.5 , and this justifies the dissimilarities among the optimal performances obtained using opt $J_{1}$, opt $J_{2} J_{7}$ and the global effects due to dynamic random simulations.

From all the previous observations, it is clear that for the case study and with the chosen initial data, the best performances on the whole network are given by the optimal algorithm for $J_{1}$. Such algorithm is preferable for maximizing traffic flows, since it allows not only the locally optimization of each node of $2 \times 1$ type, but also the global improvement 


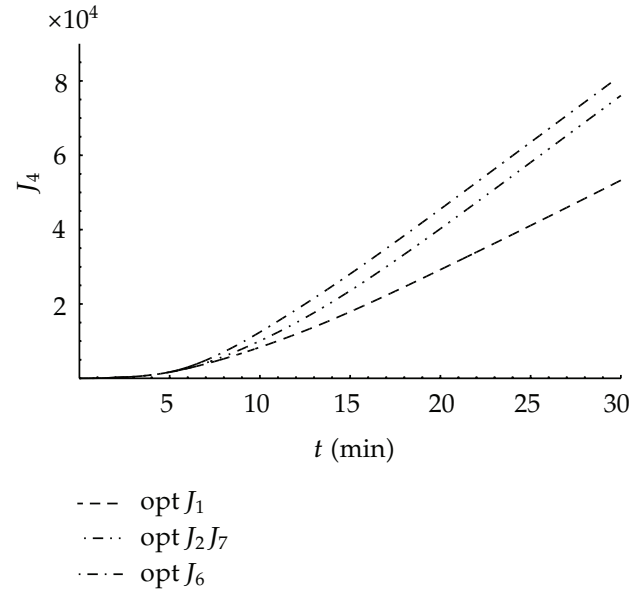

(a)

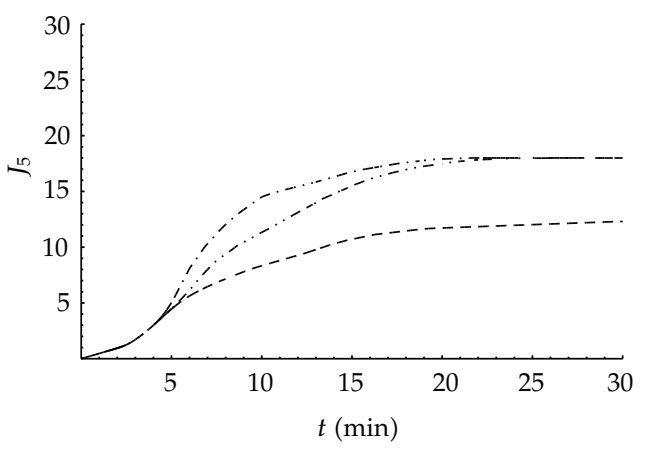

- - - opt $J_{1}$

$-\cdots$ opt $J_{2} J_{7}$

-..- opt $J_{6}$

Figure 10: Behaviour of the optimal cost functionals $J_{4}$ (a) and SGW (b).

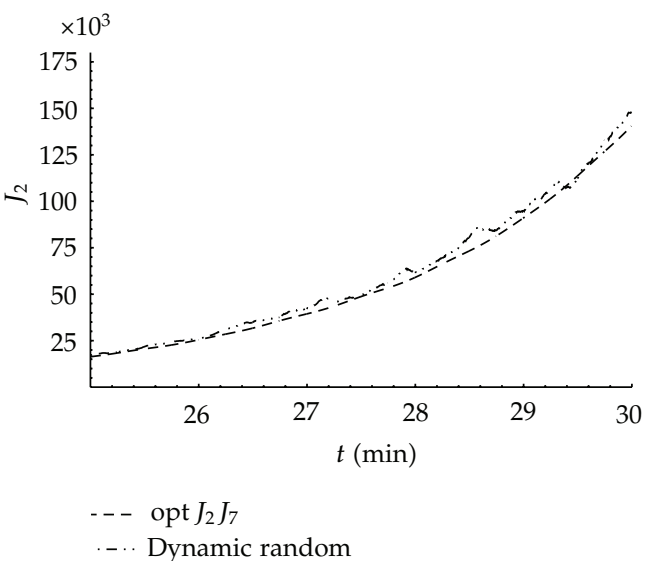

(a)

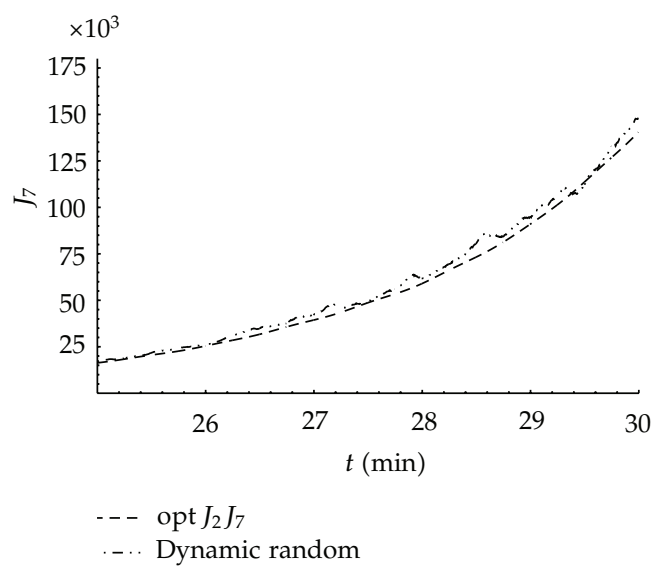

(b)

Figure 11: Behaviour of optimal $J_{2}(\mathrm{a})$ and $J_{7}(\mathrm{~b})$ versus dynamic random simulations.

of traffic conditions over the whole network. The other algorithms are able only to improve locally traffic viability.

\section{Acknowledgment}

This work is partially supported by MIUR-FIRB Integrated System for Emergency (InSyEme) project under the Grant RBIP063BPH. 


\section{References}

[1] G. M. Coclite, M. Garavello, and B. Piccoli, "Traffic flow on a road network," SIAM Journal on Mathematical Analysis, vol. 36, no. 6, pp. 1862-1886, 2005.

[2] D. Helbing, S. Lammer, and J.-P. Lebacque, "Self-organized control of irregular or perturbed network traffic," in Optimal Control and Dynamic Games, C. Deissenberg and R. F. Hartl, Eds., pp. 239-274, Springer, Dordrecht, The Netherlands, 2005.

[3] M. Gugat, M. Herty, A. Klar, and G. Leugering, "Optimal control for traffic flow networks," Journal of Optimization Theory and Applications, vol. 126, no. 3, pp. 589-616, 2005.

[4] M. Herty and A. Klar, "Modeling, simulation, and optimization of traffic flow networks," SIAM Journal on Scientific Computing, vol. 25, no. 3, pp. 1066-1087, 2003.

[5] Y. Chitour and B. Piccoli, "Traffic circles and timing of traffic lights for cars flow," Discrete and Continuous Dynamical Systems. Series B, vol. 5, no. 3, pp. 599-630, 2005.

[6] A. Cascone, C. D'Apice, P. Benedetto, and R. Luigi, "Optimization of traffic on road networks," Mathematical Models \& Methods in Applied Sciences, vol. 17, no. 10, pp. 1587-1617, 2007.

[7] A. Cascone, C. D'Apice, B. Piccoli, and L. Rarità, "Circulation of car traffic in congested urban areas," Communications in Mathematical Sciences, vol. 6, no. 3, pp. 765-784, 2008.

[8] A. Cascone, R. Manzo, B. Piccoli, and L. Rarità, "Optimization versus randomness for car traffic regulation," Physical Review E, vol. 78, no. 2, Article ID 026113, 5 pages, 2008.

[9] R. M. Colombo and A. Groli, "Minimising stop and go waves to optimise traffic flow," Applied Mathematics Letters, vol. 17, no. 6, pp. 697-701, 2004.

[10] M. J. Lighthill and G. B. Whitham, "On kinematic waves. II. A theory of traffic flow on long crowded roads," Proceedings of the Royal Society A, vol. 229, pp. 317-345, 1955.

[11] P. I. Richards, "Shock waves on the highway," Operations Research, vol. 4, pp. 42-51, 1956.

[12] M. Garavello and B. Piccoli, Traffic Flow on Networks, vol. 1 of AIMS Series on Applied Mathematics, American Institute of Mathematical Sciences (AIMS), Springfield, Mo, USA, 2006.

[13] G. Bretti, R. Natalini, and B. Piccoli, "Numerical approximations of a traffic flow model on networks," Networks and Heterogeneous Media, vol. 1, no. 1, pp. 57-84, 2006.

[14] S. K. Godunov, "A difference method for numerical calculation of discontinuous solutions of the equations of hydrodynamics," vol. 47, pp. 271-306, 1959.

[15] E. Godlewski and P.-A. Raviart, Numerical Approximation of Hyperbolic Systems of Conservation Laws, vol. 118 of Applied Mathematical Sciences, Springer, New York, NY, USA, 1996. 


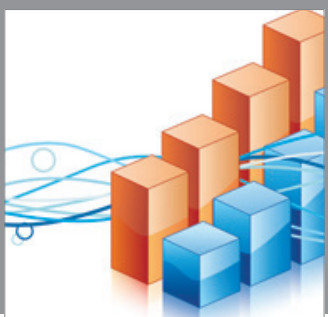

Advances in

Operations Research

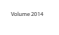

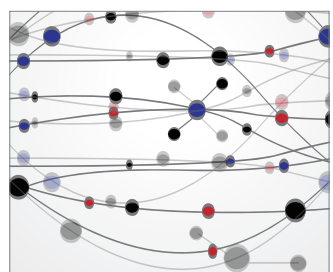

\section{The Scientific} World Journal
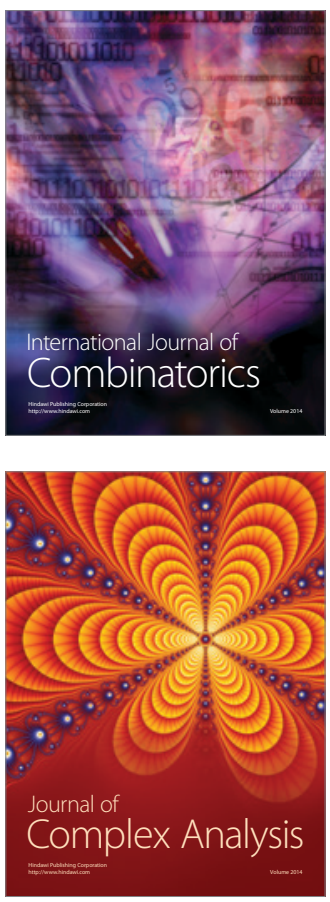

International Journal of

Mathematics and

Mathematical

Sciences
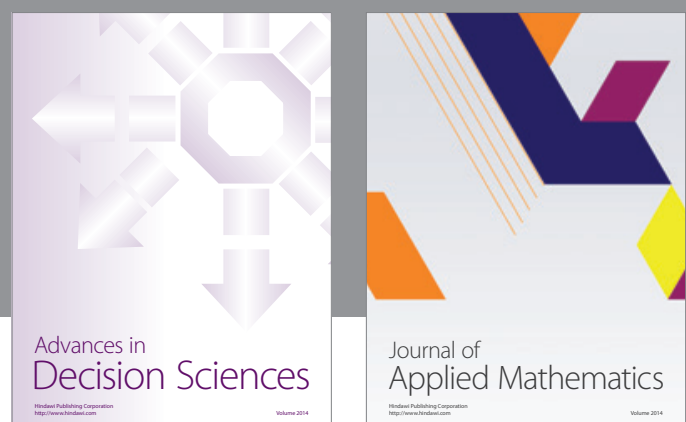

Journal of

Applied Mathematics
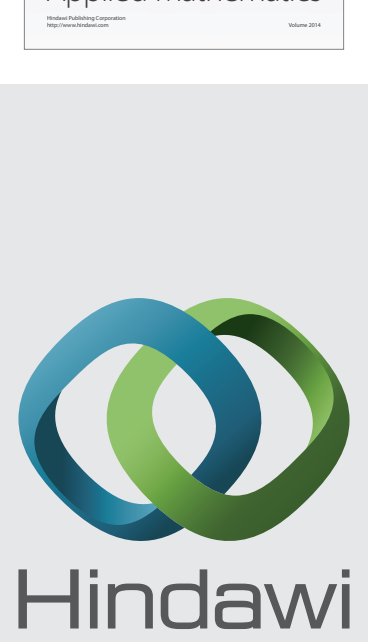

Submit your manuscripts at http://www.hindawi.com
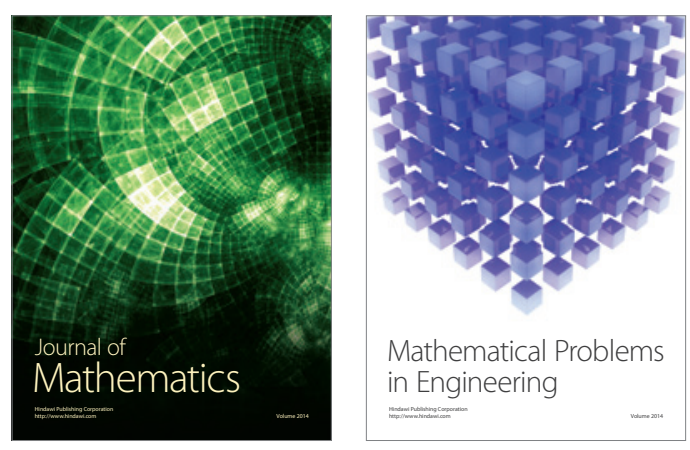

Mathematical Problems in Engineering
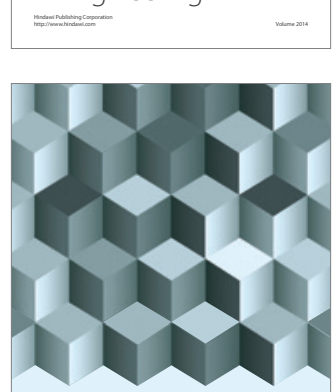

Journal of

Function Spaces
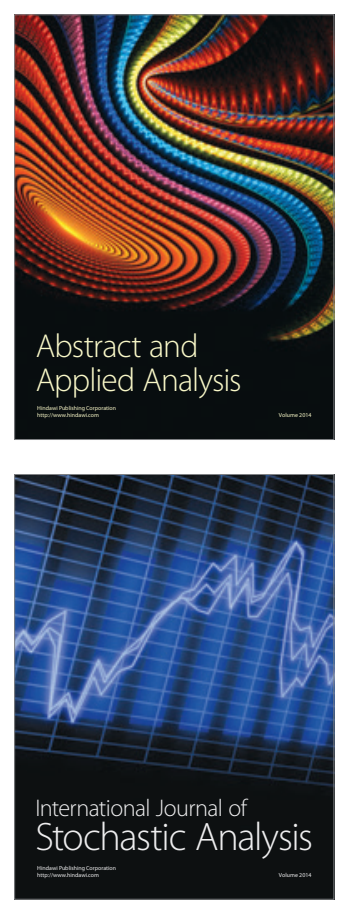

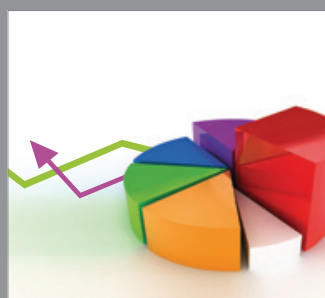

ournal of

Probability and Statistics

Promensencen
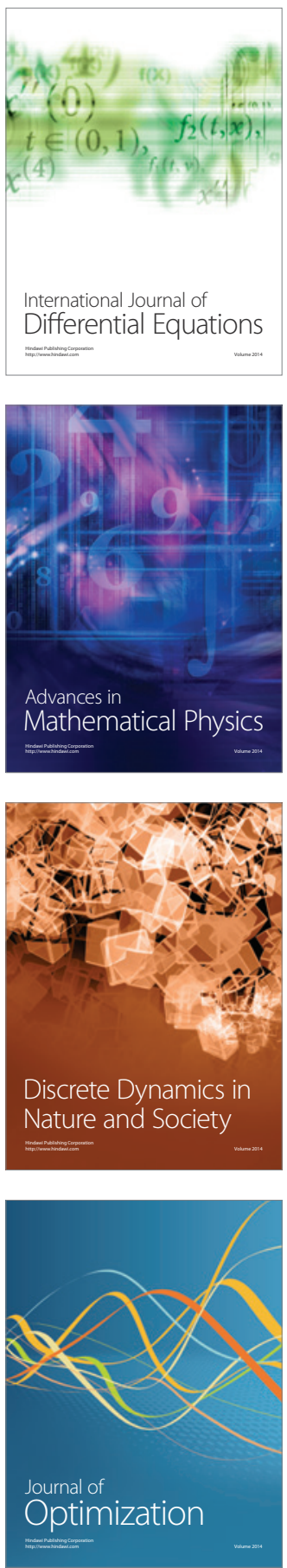